\title{
Effect of fluid shear stress on catalytic activity of biopalladium nanoparticles produced by Klebsiella Pneumoniae $\mathrm{ECU}-15$ on $\mathrm{Cr}(\mathrm{VI})$ reduction reaction
}

\author{
Bin Lei, Xu Zhang*, Minglong Zhu and Wensong Tan
}

\begin{abstract}
Background: Biopalladium (bioPd(0)) nanoparticles on Klebsiella Pneumoniae ECU-15 were synthesized mainly on the microorganism's surface. Data suggest that the resistance of mass transfer around the cell surface region plays a critical role in bioPd(0) synthesis process. However, the mechanisms for its role remains elusive.

Results: The experimental results indicated that 1) diffusion resistance existed around the microorganism's cell in reaction vessel and 2) fluid shear stress affected the mass transfer rates differently according to its strength and thus had varying effects on the bioPd(0) synthesis. More than $97.9 \pm 1.5 \%$ Chromium(VI)(Cr(VI)) $(384 \mu \mathrm{M})$ was reduced to $\mathrm{Cr}$ (III) within 20 min with $5 \% \mathrm{Pd} / \mathrm{bioPd}(0)$ as catalyst, which was generated by the K. Pneumoniae ECU-15, and the catalytic performance of $\mathrm{Pd} / \mathrm{bioPd}(0)$ was stable over 6 months. The optimal condition of bioreduction of $\mathrm{Pd}(\mathrm{II})$ to $\mathrm{Pd}(0)$ was determined at the Kolmogorov eddy length of $7.33 \pm 0.5 \mu \mathrm{m}$ and lasted for $1 \mathrm{~h}$ in the extended reduction process after the usual adsorption and reduction process.

Conclusions: It is concluded that a high bioPd(0) catalytic activity can be achieved by controlling the fluid shear stress intensity in an extended reduction process in the bioreactor.
\end{abstract}

Keywords: Biopalladium; Mass transfer resistance; Fluid shear stress; K. pneumoniae

\section{Background}

The recovery of nanopalladium particles from waste has been of great interest recently [1]. Certain physical properties of nanopalladium particles differ from those of the bulk material $[2,3]$. Palladium is used extensively because of its catalytic activity in some chemical reactions. Traditionally, the preparation of nanopalladium requires the rigorous experimental procedures. In contrast, the microbiological biosynthetic process of converting $\mathrm{Pd}(\mathrm{II})$ to $\mathrm{Pd}$ (0) works through the binding of metal $\mathrm{Pd}(\mathrm{II})$ ions onto highly reactive bacterial cell surfaces concomitant with Pd (II) ion reduction [4] at ambient temperature and pressure. This process does not require hazardous chemicals and, thus, is effective and environmentally friendly.

Many kinds of bacterial species have been used in the reduction of $\mathrm{Pd}(\mathrm{II})$ salts to their elementary metallic form [1]. Humphries et al. investigated the reduction of

\footnotetext{
* Correspondence: zhangxu@ecust.edu.cn

State Key Laboratory of Bioreactor Engineering, East China University of Science and Technology, Shanghai 200237, People's Republic of China
}

Cr(chromium(VI) by immobilized cells of Desulfovibrio vulgairs NCIMB8303 and Microbacterium sp. NCIMB 13776, which showed that the best immobilization matrices were 130 (agarose) and 15 (agar) $\mathrm{nmol} \mathrm{h}^{-1} \mathrm{mg}$ dry cell $\mathrm{wt}^{-1}$, with the highest $\mathrm{Cr}(\mathrm{VI})$ reducing efficiency [5]. Macaskie et al. studied palladium catalysts generated on gram negative (Desulfovibrio) and gram positive (Bacillus) bacterial surfaces. Discrete nanoparticles were located in the periplasmic space of Desulfovibrio desulfuricans [6] and in the peptidoglycan and proteinaceous surface layer (S-layer) of Bacillus sphaericu [7]. De Windt et al. found that the bioreductive deposition of $\operatorname{Pd}(0)$ occurred on the cell wall and in the periplasmic space of Shewanella oneidensis in the presence of a series of electron donors [8]. Deplanche et al. investigated the involvement of hydrogenase in the formation of $\operatorname{Pd}(0)$ using Escherichia coli mutant strains [9]. Martins et al. found that a Pd(II) resistant bacterial community could biorecover this metal from a solution, and that the bacterial consortium was closely related to several Clostridium species, Bacteroides, and Citrobacter [10]. Many fermentative species 
could produce hydrogen during fermentation and subsequently reduce $\mathrm{Pd}(\mathrm{II})$ to $\mathrm{Pd}(0)$. An addition of $\mathrm{Pd}(\mathrm{II})$ to the fermenting culture of Clostridium pasteurianum could result in the formation of $\operatorname{Pd}(0)$ nanoparticles on the bacterial cell wall and in the cytoplasm [11]. Klebsiella pneumoniae is a potential biohydrogen producer [12] and has the ability to bioreduce Pd(II) [13]. The evaluation of the capacity of $K$. pneumoniae to reduce $\operatorname{Pd}(\mathrm{II})$ to $\operatorname{Pd}(0)$ nanoparticles would be significant because of its respiration diversity, its wide distribution in various environments, and its potentially effective approach to the remediation of aggressive metal waste.

Biopalladium nanoparticles were synthesized mainly inside the cell surface region [14]. The resistance to mass transfer inside this region during bioPd( $(0)$ preparation should not be neglected. Han et al. found that besides the heating effect, microwaves could also enhance the mass transfer rates of active constituents. The bound potential of cell walls could be overcome by a certain microwave intensity to increase the extraction efficiency [15]. The fluid shear stress could also be used to open the periplasmic layer, reduce its mass transfer resistance and enhance the rates [16]. However, the influence of shear stress on the chemical and physical properties of biosynthetic nanopalladium particles was not fully understood. In this study, the influence of fluid shear stress during the bioPd $(0)$ preparation process on its catalytic activity for $\mathrm{Cr}(\mathrm{VI})$ reduction to $\mathrm{Cr}(\mathrm{III})$ was investigated. Physical properties of the bioPd $(0)$ were measured by a transmission electron microscopy (TEM), energy-dispersive X-ray spectroscopy (EDAX), and X-ray diffractometry (XRD). The results would be significant for the development of the efficient and stable bioPd( 0 ) catalyst production biotechnology.

\section{Methods}

\section{Preparation of the bioPd(0) catalysts}

K. pneumoniae ECU-15 was isolated from the anaerobic sewage sludge [12] and cultured to a high-cell concentration in an experimental reactor. The biomass at midlogarithmic phase cultures was harvested by a centrifuge (4000 g, $15 \mathrm{~min}, 4^{\circ} \mathrm{C}$ ). The pellets were washed three times by $100 \mathrm{~mL}$ of MOPS-NaOH buffer (20 mM, pH 7.2) and resuspended in $50 \mathrm{~mL}$ of the same buffer. And then it was stored at $4^{\circ} \mathrm{C}$ under $\mathrm{N}_{2}$ for no more than $24 \mathrm{~h}$ until use. Cell concentration $(\mathrm{mg} / \mathrm{mL})$ was determined by a correlation to a predetermined OD600 to dry weight conversion.

A known volume of the concentrated resting cell suspension was transferred anaerobically into a $100-\mathrm{mL}$ serum bottle, which contained an appropriate volume of degassed 2- $\mathrm{mM} \mathrm{PdCl}_{2}$ solution to make the final weight ratio of $\mathrm{Pd}$ to dry cells equal to 1:19. That means a final loading of $5 \%$ Pd on biomass could be obtained. A series of steps was scheduled as follows: firstly, the static adsorption process between the microorganism and the $\mathrm{Pd}(\mathrm{II})$ lasted for
$30 \mathrm{~min}$, then the solution was sparged by $\mathrm{H}_{2}$ for $20 \mathrm{~min}$ in the reduction process. The $\mathrm{H}_{2}$ was retained in the serum bottle till the end of the preparation process. The degree of the $\mathrm{Pd}(\mathrm{II})$ reduction from the solution was confirmed by assaying the residual $\mathrm{Pd}(\mathrm{II})$ ion concentration by the $\mathrm{SnCl}_{2}$ method in cell/Pd mixture supernatant [17]. The $\mathrm{Pd}(0)$ coated biomass was harvested by centrifugation $(4,000 \mathrm{~g}$, $15 \mathrm{~min}, 25^{\circ} \mathrm{C}$ ) and rinsed three times by a distilled water $\left(\mathrm{dH}_{2} \mathrm{O}\right)$. The black precipitate was washed once by acetone and left to dry in air for overnight to a constant weight and finely ground in a mortar. The physical properties of the Pd-loaded bacterial cells were measured using TEM (JEM2100, JEOL, Akishima-shi, Japan), EDAX (DX-4, EDAX, Mahwah, NJ, USA), and the XRD (D/max2550B/PC, Rigaku, Shibuya-ku, Japan). The catalytic activity of the bioPd $(0)$ was tested without further processing.

\section{Transmission electron microscopy}

The bacteria loaded with the palladium were rinsed twice with the distilled water and then fixed in the $2.5 \%$ glutaraldehyde. After being centrifuged at 10,000 rpm, the pellets of palladium-loaded bacteria were resuspended in $15 \mathrm{~mL}$ of $0.1 \mathrm{M} \mathrm{Na}$-cacodylate buffer and then stained for an hour in the $1 \%$ osmium tetroxide solution in $0.1 \mathrm{M}$ phosphate buffer at $\mathrm{pH} 7$ for the measurement of the TEM. Cells were dehydrated for $15 \mathrm{~min}$ by the $70 \%, 90 \%$, and $100 \%$ ethanol solution, respectively. Then, it was washed twice in the propylene oxide for $15 \mathrm{~min}$. The cells were embedded in the epoxy resin and then the mixture was left to be polymerized at $60^{\circ} \mathrm{C}$ for $24 \mathrm{~h}$. A section (100-nm thick) was cut from the resin block, placed onto a copper grid, and then viewed with TEM (JEM-2100, JEOL, Akishima-shi, Japan).

\section{EDAX and XRD measurements}

For the EDAX and the XRD analysis, the parallel samples of $\operatorname{Pd}(0)$-loaded biomass were washed for three times in the distilled water and once in acetone. After the centrifugation process, the $\operatorname{Pd}(0)$-loaded biomass were resuspended in a small volume of acetone and then air-dried to a constant weight. Finally, it was grounded to a fine powder by an agate mortar. The powder was analyzed using the EDAX (DX-4, EDAX, Mahwah, NJ, USA) and the XRD (D/max2550B/PC, Rigaku, Shibuya-ku, Japan). The powder pattern was compared to the references in the Joint Committee for Powder Diffraction Studies (JCPDS) database.

\section{Evaluation of catalytic activity via $\mathrm{Cr}(\mathrm{VI})$ reduction to $\mathrm{Cr}(\mathrm{III})$}

The bioPd $(0)$ could be used as the catalyst for the reduction of $\mathrm{Cr}(\mathrm{VI})$ to $\mathrm{Cr}(\mathrm{III})$. In this paper, a comparative study of the catalytic activity of bioPd(0) under various condition of preparation was carried out. During the experimental process, $10 \mathrm{mg} \operatorname{bioPd}(0)$ were accurately 
weighed and then put into a $25-\mathrm{mL}$ serum bottle sealed with butyl rubber stopper, containing $10 \mathrm{~mL}, 384 \mu \mathrm{M} \mathrm{Cr}$ (VI). The mixture was made anaerobic by sparging the solution with oxygen free nitrogen for $10 \mathrm{~min}$. The bottle was stalled in a $30^{\circ} \mathrm{C}$ water bath for $30 \mathrm{~min}$ before the reaction. The reaction was initiated when $1 \mathrm{~mL}$ of sodium formate as the electron donor was added with the final concentration to $25 \mathrm{mM}$. The samples were periodically withdrawn from the bottle via the rubber septa and centrifuged at $4,000 \mathrm{rpm}$ for $2 \mathrm{~min}$ to remove the bioPd $(0)$ catalyst. The supernatant was measured to determine the concentration of $\mathrm{Cr}(\mathrm{VI})$ and $\mathrm{Cr}(\mathrm{III})$.

\section{Effect of different conditions on catalytic activity in the extended reduction of $\mathrm{Cr}(\mathrm{VI})$ to $\mathrm{Cr}(\mathrm{III})$}

Comparative studies of the catalytic activity of bioPd(0) for $\mathrm{Cr}(\mathrm{VI})$ reduction to $\mathrm{Cr}(\mathrm{III})$ were carried out under different bioPd $(0)$ preparation conditions. The preparation of bioPd (0) nanoparticles through the reduction of $\mathrm{Pd}(\mathrm{II})$ by $K$. pneumoniae ECU-15 comprised three steps, as represented schematically in Figure 1. In the adsorption step, the $\mathrm{Pd}(\mathrm{II})$ ions in the solution were adsorbed on the cell surface. In the reduction step, the adsorbed $\mathrm{Pd}(\mathrm{II})$ ions were reduced to $\operatorname{Pd}(0)$ at the cell surface with hydrogen sparging. And in the third step, the reduction process was extended through the introduction of stationary or shaking factors, which represented the new findings of this paper.

Stationary operation in the extended reduction process referred to the cell $/ \operatorname{Pd}(0) / \operatorname{Pd}(\mathrm{II})$ mixture solution being allowed to stand from 0 to $13 \mathrm{~h}$. $\operatorname{BioPd}(0)$ was harvested and used as $\mathrm{Cr}(\mathrm{VI})$ reduction catalyst after several hours of standing time. The variation in shaking factors referred to a variation in shaker speed from 50 to $200 \mathrm{rpm}$ and time from 1 to $3 \mathrm{~h}$ only after hydrogen sparging. The reduction of $\mathrm{Cr}(\mathrm{VI})$ was estimated as the percent of $\mathrm{Cr}(\mathrm{VI})$ which were reduced to $\mathrm{Cr}(\mathrm{III})$ at the set time. The reduction ratio could be calculated from the following formulas:

$$
\eta=\frac{C_{0}-C_{t}}{C_{0}}
$$

Where $C_{0}$ was the initial concentration of $\mathrm{Cr}(\mathrm{VI})$, and $C_{\mathrm{t}}$ was the concentration of $\mathrm{Cr}(\mathrm{VI})$ at time $t$.

\section{Results and discussion}

Examination of bioPd(0) samples by electron microscopy, energy dispersive X-ray spectroscopy, and X-ray diffraction As shown in Figure 2, the color of the solution in the reduction system changed from milk white to black, which indicated that the $\operatorname{Pd}(0)$ might be generated through the reduction of $\mathrm{Pd}(\mathrm{II})$ ions by the $K$. pneumoniae ECU-15. After the centrifugation and rinse treatment, the black precipitate was dried to a constant weight and finely ground in a mortar, which was finally stored in the vacuum desiccator.

The TEM photographs of the Pd-located cells of the K. pneumoniae ECU-15 were shown in Figure 3a,b,c,d. The nanoPd(0) deposits were almost the round black particles with similar morphology. It was also shown that the Pd particles were distributed on the periplasmic space and the cytoderm of the $K$. pneumoniae ECU-15, which was consistent with that of the previous research [18]. It seems that there was no bioPd(0) appeared in the intercellular space, which indicated that the nano bioPd(0) might be generated by some catalytic reduction reaction on the cell surface of $K$. pneumoniae ECU-15. According to the various magnifications of the TEM images of the bioPd(0) nanoparticals, it seems that the particles size ranged from 5.0 to $26.5 \mathrm{~nm}$, with the average article size of $15.8 \mathrm{~nm}$. The identity of the nanoparticle deposits as bioPd(0) was confirmed by the EDAX analysis shown in Figure 3e, where the Pd components was obvious. The $\mathrm{C}, \mathrm{O}$, and $\mathrm{P}$ components shown in Figure $3 e$ were the cellular material. The characteristic diffraction peak of the crystalline $\operatorname{Pd}(0)$ particles at $2 \theta=40^{\circ}, 47^{\circ}$, and $68^{\circ}$ were also shown by the XRD diffractograms, as reported by Hennebel et al. [13]. It was also confirmed that the cell-mediated reduction of the $\mathrm{Pd}(\mathrm{II})$ to the metallic $\operatorname{Pd}(0)$, with a face-centered cubic structure $[19,20]$ through the XRD patterns of the $\mathrm{Pd}(\mathrm{II})$ challenged cells, were shown in Figure 3f. All these results proved that the generation of the bioPd(0) was through the reduction of the $\mathrm{Pd}(\mathrm{II})$ to $\mathrm{Pd}(0)$ on the surface of the K. pneumoniae ECU-15 cells. The location of the bioPd( 0 ) nanoparticles generated might be caused by the distribution and properties of hydrogenase and other enzymes involved in $\mathrm{Pd}(\mathrm{II})$ reduction [11]. However, Rotaru found that the cell surface functional groups really played a

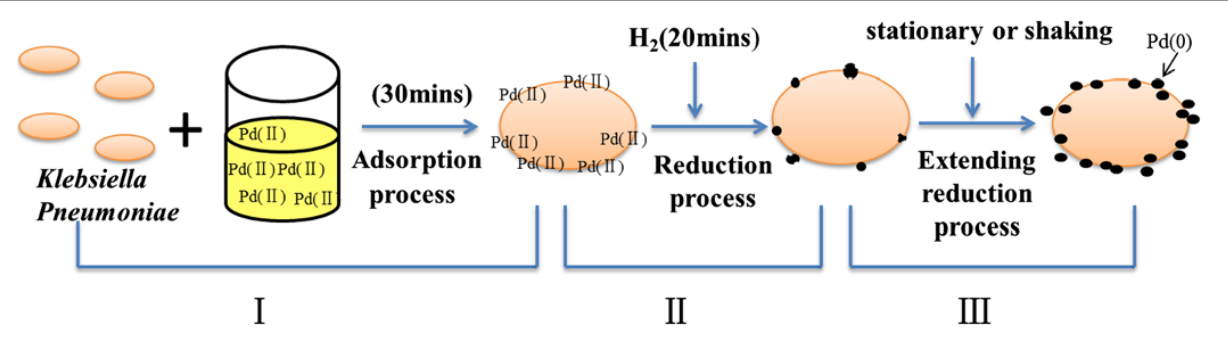

Figure 1 Schematic representation of the bioPd(0) synthesis. 


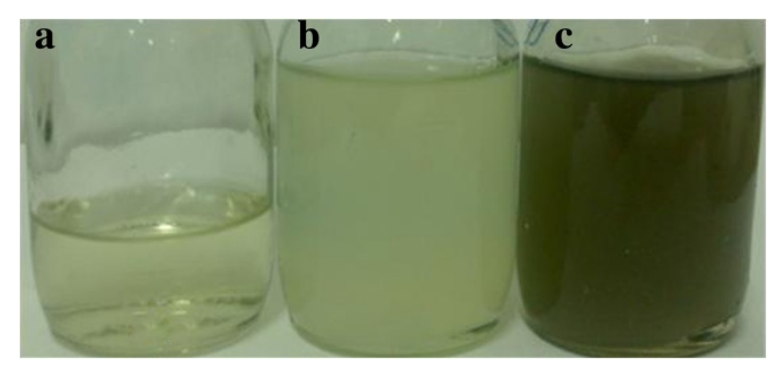

Figure 2 Photographs of color during the process of $\mathrm{Pd}(\mathrm{II})$ reduction to $\operatorname{Pd}(0)$ with $K$. pneumoniae ECU-15. (a) $\mathrm{Pd}(\mathrm{II})$ solution, (b) $\mathrm{Pd}(\mathrm{II})$ and the K. pneumoniae suspension, and (c) bioPd(0) and the K. pneumoniae solution.

catalytic function role for the $\mathrm{Pd}(\mathrm{II})$ reduction to the $\operatorname{bioPd}(0)$ [3]. These mechanisms were not yet unified and needed to be explored carefully. The accepted view was that the reactant should firstly transfer from the solution to the function groups or the enzymes on the cell surface, and thus, the resistance of mass transfer inside the cell surface scaffold should not be neglected.

\section{Effect of extended reduction standing time during bioPd (0) preparation on catalytic activity for $\mathrm{Cr}(\mathrm{VI})$ reduction to $\mathrm{Cr}$ (III)}

The cell wall of $K$. pneumoniae ECU-15 is composed mainly of peptidoglycan, which contributes to the mechanical strength of the bacterial cell structure and prevents the intrusion of various ions as a barrier to mass transfer [21]. As discussed above, the synthesis process of bioPd(0) nanoparticles by the reduction of Pd(II) on $K$. pneumoniae ECU-15 included an adsorption process and a reduction process [20]. In order to confirm the time of the adsorption process, the adsorption efficiency of $\mathrm{Pd}(\mathrm{II})$ in the solution could be determined by detecting the residual $\mathrm{Pd}(\mathrm{II})$ concentration through the $\mathrm{SnCl}_{2}$ method. The preliminary experiments showed that the residual $\mathrm{Pd}(\mathrm{II})$ concentration gradually decreased within the first $30 \mathrm{~min}$, and the adsorption equilibration of $\mathrm{Pd}$ (II) would be reached at $30 \mathrm{~min}$. The influence of standing time on the extended reduction of $\mathrm{Cr}(\mathrm{VI})$ to $\mathrm{Cr}(\mathrm{III})$ by bioPd $(0)$ catalytic activity was given in Figure 4 . The catalytic activity was improved by extending the reduction time. An increase in standing time from 0 to $6 \mathrm{~h}$ resulted in the complete reduction of $\mathrm{Cr}(\mathrm{VI})$ to $\mathrm{Cr}(\mathrm{III})$ in $1 \mathrm{~h}$ instead of $5 \mathrm{~h}$. Especially, the $\mathrm{Cr}(\mathrm{VI})$ reduction ratio $(\eta)$ has increased exponentially in the first $1 \mathrm{~h}$ with the standing treatment. With a further increase in standing time, the improvement in catalytic activity of the bioPd (0) was not obvious. Extending the reduction time would influence the equilibrium time for bioPd(0) generation, which influence the bioPd(0) catalytic activity indirectly. The optimal standing time was found to be $6 \mathrm{~h}$. The TEM micrograph in Figure 5 showed that the nanoparticles were distributed homogeneously with higher density in the periplasmic space and cytoderm with the longer standing time (Figure 5a,c), while in Figure 5b,d, the palladium particles demonstrated with the properties of uneven size and inhomogeneity distribution. This observation further confirmed the results in Figure 4.

During the $\operatorname{Pd}(\mathrm{II})$ reduction process, the $\mathrm{Pd}(\mathrm{II})$ ions and molecular hydrogen in the solution would pass through several layers, such as the gas-liquid, liquidliquid, and solid-liquid layers before arriving at the hydrogenase and functional organic groups on the membrane and periplasmic space of the cells [22]. It was followed by the nucleation of $\operatorname{Pd}(0)$ and further cluster growth. The three-dimensional structure of the periplasmic space around the bacterial cell had a certain resistance to the ions [23] and the molecular diffusion process and more time was required for the delivery of $\mathrm{Pd}(\mathrm{II})$ ions and molecular hydrogen to the microorganism cell surface to complete the reduction of $\operatorname{Pd}(\mathrm{II})$ to $\mathrm{Pd}(0)$. Thus, it's necessary to overcome the resistance to mass transfer around the microgram cell for the efficient preparation of stable and high efficiency bioPd(0).

\section{Effect of fluid shear stress during Pd(II) adsorption and reduction processes on the catalytic activity of bioPd(0) for $\mathrm{Cr}(\mathrm{VI})$ reduction to $\mathrm{Cr}(\mathrm{III})$}

It is critical that the $\mathrm{Pd}(\mathrm{II})$ ion and molecular hydrogen were transferred efficiently to the synthesis site of the bioPd(0) on the cell surface of $K$. pneumoniae ECU-15. Therefore, mass transfer problems in the preparation of bioPd(0) should be investigated. The fluid shear stress, which was generated by various flask shaking intensities, could reduce the mass transfer resistance in the periplasmic layer $[23,24]$. The movement of the turbulent eddies, which were produced by fluid turbulence intensities, would generate fluid shear stress [25]. The turbulence intensity could be demonstrated by the Kolmogorov eddy length of the fluid [26]. For an internal fluid in the Erlenmeyer flask at different shaking speeds, the Kolmogorov eddy length of the fluid turbulence could be calculated from Equation (2) [27]. The estimated values for the energy dissipation rate and smallest eddy size at different shaking speeds were given in Table 1 . When the shaking speeds increased from 50 to $200 \mathrm{rpm}$, the Kolmogorov eddy length would decrease from 160.69 to $3.26 \mu \mathrm{m}$. And thus, the impact of fluid shear force could be expressed quantitatively by the Kolmogorov eddy length.

$$
\lambda=\left(\frac{\nu^{3}}{\varepsilon}\right)^{0.25}, \quad \varepsilon=k \rho^{-1} V_{t}^{-0.25} N^{2.81}
$$

where $\lambda$ is the Kolmogorov eddy length $(\mu \mathrm{m}), v$ is the fluid kinematic viscosity $\left(\mathrm{m}^{2} / \mathrm{s}\right), \varepsilon$ is the unit average 

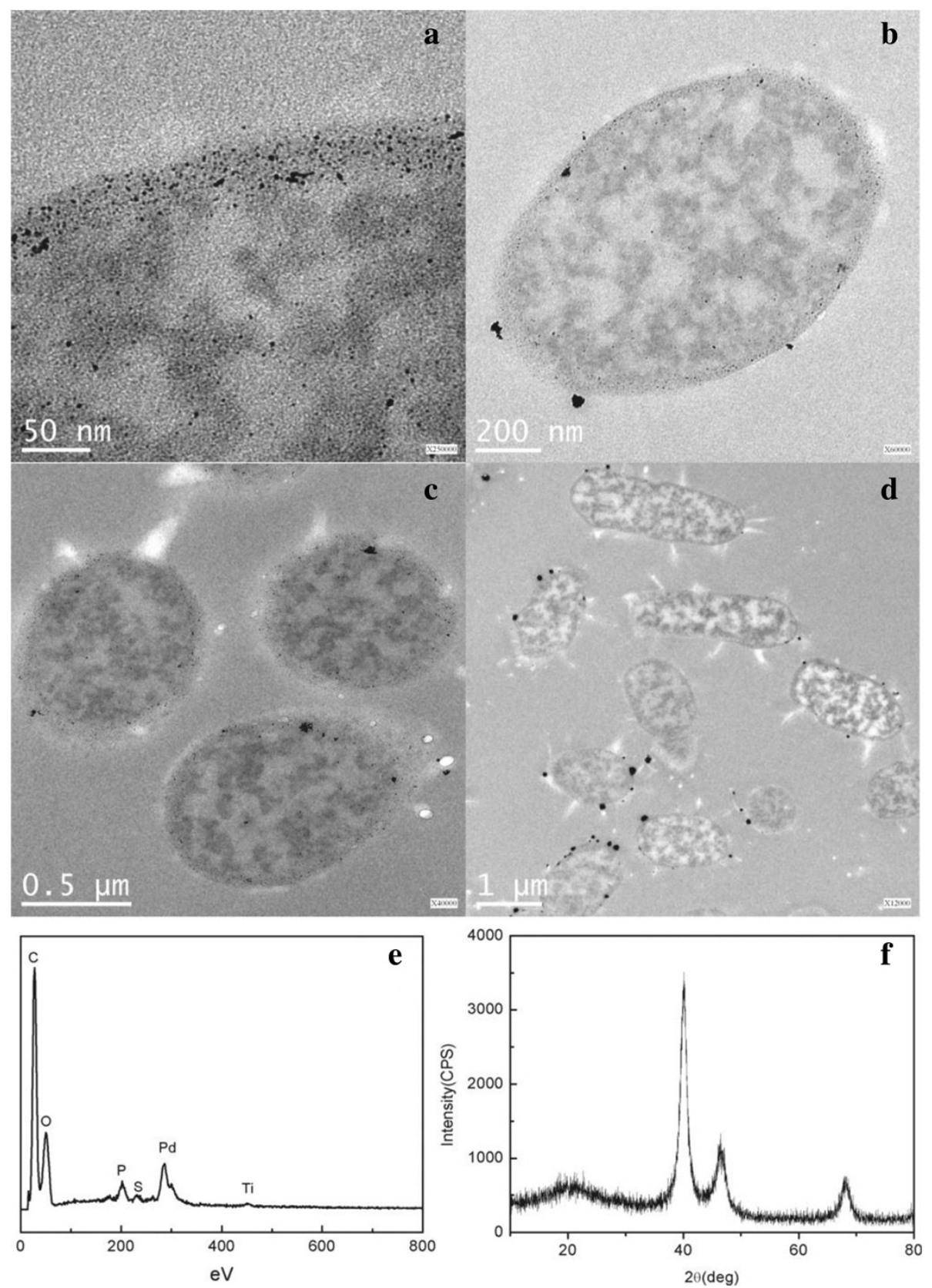

Figure 3 Properties of bioPd(0) generated by Pd(II) reduction on the cell surface of the $K$. pneumoniae ECU-15. (a-d) The TEM of nanoparticles of $\mathrm{Pd}(0)$ at different scale; (e) The EDAX results of $\mathrm{Pd}(0)$; (f) The XRD patterns of $\mathrm{Pd}(0)$.

energy consumption power of the Erlenmeyer flask, $k$ is the constant $\left(1.09 \times 10^{-3}\right), \rho$ is the liquid density $\left(\mathrm{kg} / \mathrm{m}^{3}\right)$, $V_{t}$ is the working volume in the Erlenmeyer flask $\left(\mathrm{m}^{3}\right)$, and $N$ is the shaking speed of the Erlenmeyer flask $\left(\mathrm{s}^{-1}\right)$.

Additional file 1: Figure S1 showed the recovery ratios of $\operatorname{Pd}(0)$ at different shaking speed. It seems that all the recovery of $\operatorname{Pd}(0)$ exceeded $90 \%$ with little difference shown among the various shaking speeds. The possible reason was that the $\mathrm{Pd}(\mathrm{II})$ was mainly reduced by $\mathrm{H}_{2}$, but the other factors, such as the fluid shear stress, the hydrogenase, etc., in the bioreduction process predominantly regulated and controled the physical properties of the $\operatorname{Pd}(0)$, which fundamentality influenced the catalytic activity of $\operatorname{Pd}(0)$. The effects of shaking speed during the adsorption and the reduction process on the catalytic activity of bioPd(0) for $\mathrm{Cr}(\mathrm{VI})$ reduction to $\mathrm{Cr}$ (III) were studied in this study with results shown in Figure 6a. The $\eta$ of the $\mathrm{Cr}(\mathrm{VI})$ reduction to $\mathrm{Cr}$ (III) increased as the reaction time increased, and gradually added to a certain value. At the initial time of the reduction process, the 


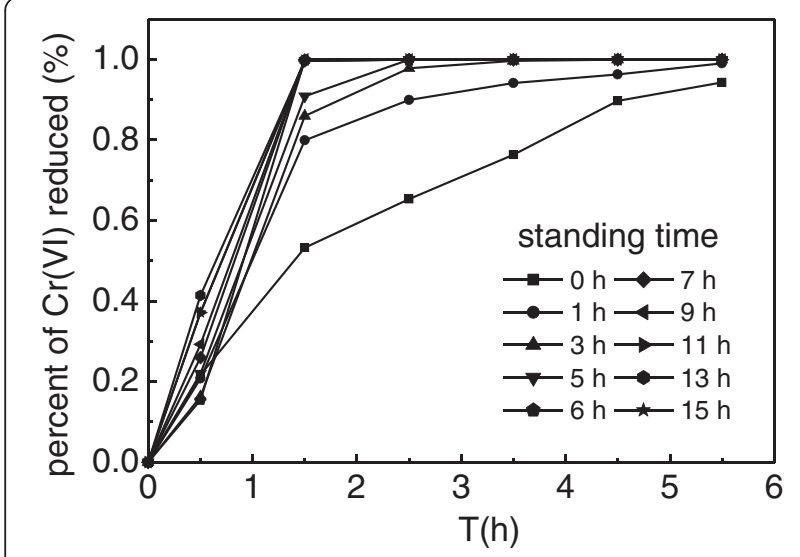

Figure 4 Effect of the standing time in the extending reduction process on the catalytic activity of bioPd(0).

reduced rates of $\mathrm{Cr}(\mathrm{VI})$ to $\mathrm{Cr}(\mathrm{III})$ increased exponentially by the bioPd $(0)$ generated with the shaking treatment, which was obviously higher than those without shaking. After $3.5 \mathrm{~h}, 84.6 \% \mathrm{Cr}(\mathrm{VI})$ was reduced to $\mathrm{Cr}(\mathrm{III})$ by the bioPd $(0)$ generated at the shaking speed of $150 \mathrm{rpm}$. It reached the $95 \%$ with the bioPd $(0)$ prepared at $200 \mathrm{rpm}$, while only $60 \% \mathrm{Cr}(\mathrm{VI})$ was reduced with $300 \mathrm{rpm}$. It was indicated that the higher shear stress was beneficial to the synthesis of bioPd(0) with higher catalytic activity. However, too high shear stress, such as $300 \mathrm{rpm}$, would be harmful, which adversely affect the preparation of bioPd (0). Approximately $5.5 \mathrm{~h}$ was required for the accomplishment of the reduction of $\mathrm{Cr}(\mathrm{VI})$ to $\mathrm{Cr}(\mathrm{III})$ by the bioPd( $(0)$ generated at a shaking speed of 150 or $200 \mathrm{rpm}$ in the generated process. The data suggested that the shaking during adsorption or reduction process did not obviously impact on the improvements in the catalytic activity of the bioPd (0). It was estimated that this process was reaction controlled, and the increase of the mass transfer rates would not visibly influence the bioPd(0) catalytic activity.

\section{Effect of fluid shear stress during the extended Pd(II) reduction process on catalytic activity of bioPd(0) for $\mathrm{Cr}(\mathrm{VI})$ reduction to $\mathrm{Cr}(\mathrm{III})$}

The cell-Pd(II) mixed with liquor after 30 min adsorption was sparged with hydrogen for 20 min under static conditions before it was placed on a shaking table. As seen in Figure $6 \mathrm{~b}$, the $\eta$ was studied as functions of the different shaking speeds. The catalytic activity of bioPd (0) on the $\eta$ could be improved severalfold when the shaking speeds were increased from 50 to $200 \mathrm{rpm}$. The optimum reduction condition was determined at $75 \mathrm{~min}$, $150 \mathrm{rpm}$. When the shaking speeds increased from 50 to $150 \mathrm{rpm}$, the catalytic activity of bioPd(0) increased proportionately. However, the catalytic activity of the bioPd (0) decreased at $200 \mathrm{rpm}$. The catalytic activity of bioPd (0) with shear stress was higher than that without. And then the influence of the shaking time on the catalytic activity in the extended reduction process was investigated. As seen in Additional file 1: Figure S2, there was no obvious improvement in the catalytic activity of bioPd(0) with the time extended. The effect of fluid shear stress on bioPd $(0)$ catalytic activity varied among the adsorption,

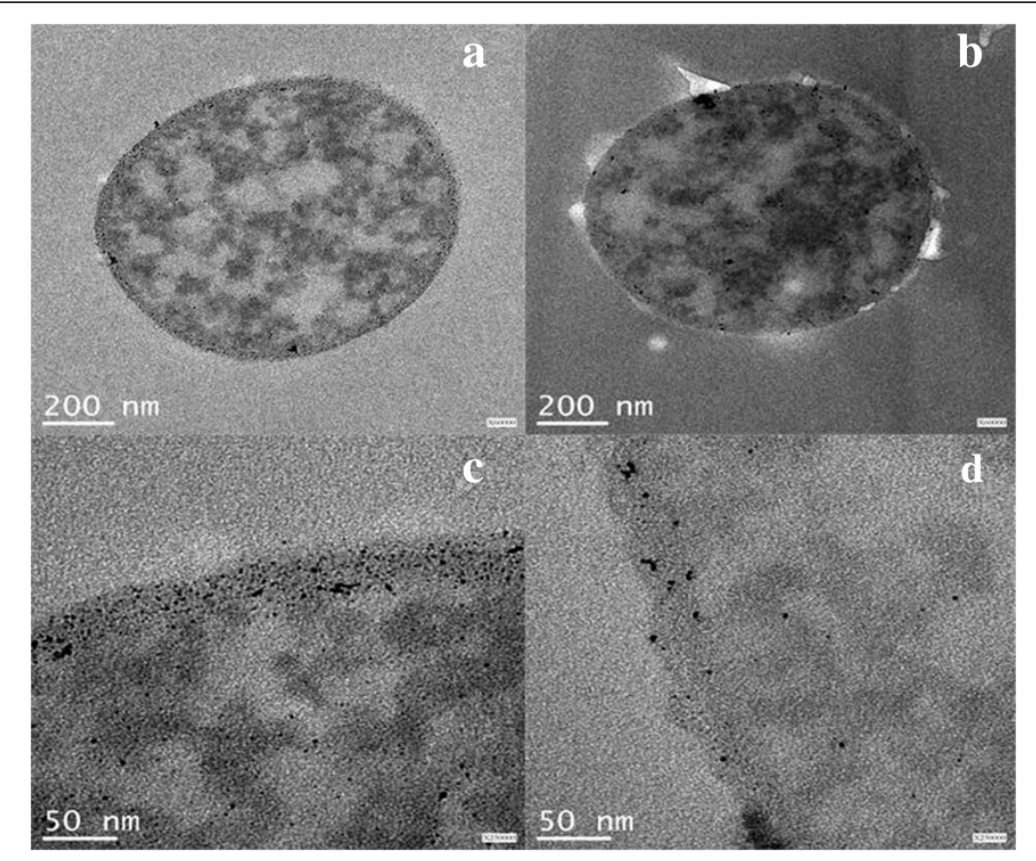

Figure 5 TEM photo of bioPd(0) at different standing time in the extending reduction process. TEM photo of bioPd( $(0)$ collected at a standing time of $6 \mathrm{~h}(\mathbf{a}, \mathbf{c})$ and collected immediately $(\mathbf{b}, \mathbf{d})$ in the extending reduction process. 
Table 1 Estimated values for energy dissipation rate and smallest eddy size of the reactor fitted at different speeds

\begin{tabular}{lcc}
\hline Shaking speeds $(\mathbf{r p m})$ & $\boldsymbol{\varepsilon}\left(\boldsymbol{W} / \mathbf{K g}\right.$ or $\left.\mathbf{~}^{\mathbf{2}} \mathbf{s}^{\mathbf{3}}\right)$ & $\boldsymbol{\lambda}(\boldsymbol{\mu m})$ \\
\hline 50 & 0.006 & 160.69 \\
100 & 0.044 & 22.91 \\
150 & 0.138 & 7.33 \\
200 & 0.312 & 3.26 \\
\hline
\end{tabular}

reduction, and extended reduction process. In the adsorption and reduction processes, more time was required for the dissolved hydrogen molecules to reach a certain concentration around the cell surface before the beginning of the bioPd( 0 ) synthesized process; whereas in the extended reduction process, when the dissolved hydrogen molecule and $\mathrm{Pd}(\mathrm{II})$ concentrations in the periplasmic region reached a certain level, strengthening the mass transfer for generating biological palladium would have a significant effect on the catalytic activity of bioPd $(0)$. Hence, it was speculated that the shaking might assist in the even dispersion of $\mathrm{Pd}(\mathrm{II})$ on or into the location of $\operatorname{Pd}(0)$ generated sites. The fluid shear stress in the extended reduction process could affect the catalytic activity of bioPd(0) significantly. A possible explanation was that this stage was mass transfer controlled, and strengthening the mass transfer would be beneficial to generate bioPd $(0)$ with high catalytic activities [28]. It required the further research.

The diffusion rates of $\mathrm{Pd}(\mathrm{II})$ ions and hydrogen molecules from the solution to the liquid boundary layer surrounding the bacterial cell increased with fluid turbulence intensities. The thickness of the liquid boundary layer and the external diffusion resistance decreased with turbulence intensity and fluid shear stress [29]. The too high shear stress might reduce the enzyme activity and destroy the cellular structure $[24,30]$. Small eddies, similar in size to those of the cells, could cause the higher shear stresses on the cells and lead to the physical damage [31]. As shown in Table 1, when the shaking speeds increased from 50 to $150 \mathrm{rpm}$, the smallest eddy sizes decreased from 160.69 to $7.33 \mu \mathrm{m}$, which was greater than the size of the K. pneumoniae ECU-15 cells $(1-1.5 \mu \mathrm{m})$. However, when the shaking speed was $200 \mathrm{rpm}$, the smallest eddy size $(3.26 \mu \mathrm{m})$ was close to that of the $K$. pneumoniae ECU-15 cells with the catalytic activity of the bioPd(0) for $\mathrm{Cr}(\mathrm{VI})$ reduction to $\mathrm{Cr}$ (III) decreased. A suitable fluid shear stress would probably decrease the mass transfer resistance with more bioPd(0) being nucleated and evenly distributed on the cells. Considering the time consumption and cost of resources, the optimal conditions for the preparation of high catalytic activity bioPd $(0)$ were to shake the mixed liquor at $150 \mathrm{rpm}$ during the extended reduction process.
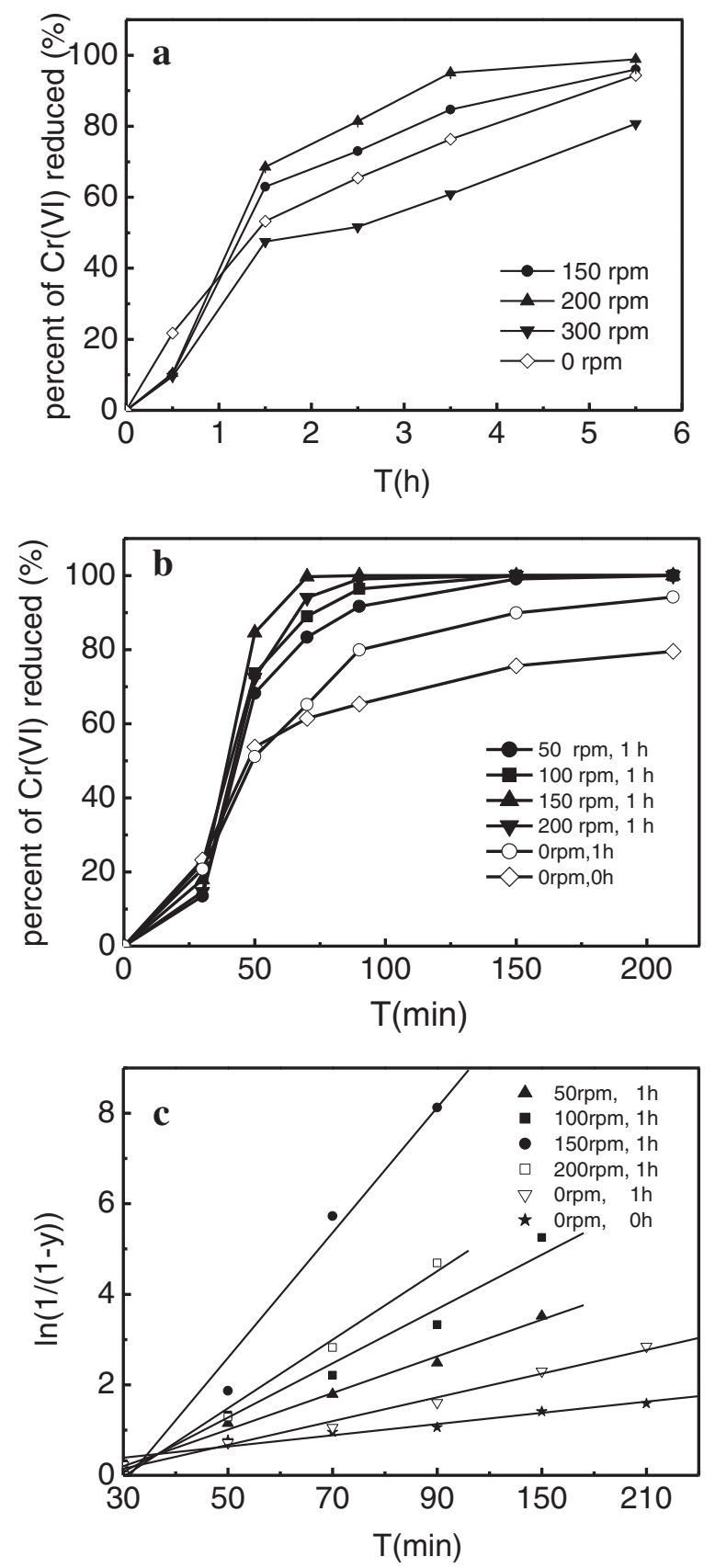

Figure 6 The effects of fluid shear stress in the preparation to the catalytic activity of bioPd(0). The effect of the shaking in adsorption process and reduction process to catalytic activity of bioPd(0) (a). The effect of the shaking speed and the shaking time in the extending reduction process to the conversion of the reduction of $\mathrm{Cr}(\mathrm{VI})$ to $\mathrm{Cr}(\mathrm{III})$ with the bioPd(0) as catalyst (b). The constant of reaction rate of bioPd(0)s on the reduction of $\mathrm{Cr}(\mathrm{VI})$ to $\mathrm{Cr}($ III) at different shaking speeds (c)

More than $97.9 \pm 1.5 \% \mathrm{Cr}(\mathrm{VI})(384 \mu \mathrm{M})$ was reduced to $\mathrm{Cr}(\mathrm{III})$ within $20 \mathrm{~min}$ with $5 \% \mathrm{Pd} / \mathrm{bioPd}(0)$ as the catalyst. The catalytic performance of the bioPd(0) was stable over 6 months. The $5 \% \mathrm{Pd} /$ carbon commercial catalyst 
could reduce $96 \pm 1.3 \% \mathrm{Cr}(\mathrm{VI})(500 \mu \mathrm{M})$ in $60 \mathrm{~min}$ [9]. It seems that the bioPd $(0)$ generated from the fluid shear stress regulation process could be a competitive biocatalyst.

The $\mathrm{Cr}(\mathrm{VI})$ reaction ratio changed with different types of bioPd $(0)$ catalyst and was solely attributed to the different shaking speeds in the extended reduction process. The reaction ratio constants $(k)$ of $\mathrm{Cr}(\mathrm{VI})$ to $\mathrm{Cr}(\mathrm{III})$ with different types of bioPd $(0)$ as catalysts were calculated from Equation (3). The slope of the lines in Figure 6c was the reaction ratio constant $(k)$. A maximum $k$ of $1.199 \mathrm{~min}^{-1}$ was obtained at $150 \mathrm{rpm}$. The difference in maximum $k$ was mainly because of the maximum catalytic activity of the bioPd( $(0)$, which resulted from the different shaking speeds in the extended reduction process. A suitable shaking speed would reduce the reaction time of $\mathrm{Cr}(\mathrm{VI})$ to $\mathrm{Cr}(\mathrm{III})$ reduction process.

$$
\operatorname{In}\left(\frac{1}{1-y}\right)=k t
$$

where $y$ is the percentage $\mathrm{Cr}(\mathrm{VI})$ consumption, $t$ is the test time (s), and $k$ is the reaction rate constant.

\section{Conclusions}

$\operatorname{BioPd}(0)$ was produced inside the periplasm of $K$. pneumoniae ECU-15 cells and could catalyze the reduction of $\mathrm{Cr}(\mathrm{VI})$ to $\mathrm{Cr}(\mathrm{III})$. Extending the standing time in the reduction process would be benefical for the biosynthesis of the bioPd(0) with high catalytic activity. Increasing the mass transfer rates through regulating the fluid shear stress for the extended reduction process during the bioPd (0) generation process would improve the catalytic activity of bioPd(0) for the reduction of $\mathrm{Cr}(\mathrm{VI})$ to $\mathrm{Cr}(\mathrm{III})$. The optimal condition of the bioreduction of $\operatorname{Pd}(\mathrm{II})$ to $\operatorname{Pd}(0)$ was at the Kolmogorov eddy length of $7.33 \pm 0.5 \mu \mathrm{m}, 1 \mathrm{~h}$ in the extended reduction process after the usual adsorption and reduction process. Increasing the mass transfer rate by the regulation of the fluid shear stress could provide certain advantages and possibilities for the development of bioreactor technologies for the noble metal recovery.

\section{Additional file}

Additional file 1: The supplementary information. The bioreduced of $\mathrm{Pd}(\mathrm{II})$ as the function of the shaking speed (Figure S1). The effect of rotating speed with different time to the catalytic activity of BioPd(0) (Figure S2).

\section{Competing interests}

The authors declare that they have no competing interests.

\section{Authors' contributions}

$\mathrm{BL}, \mathrm{XZ}, \mathrm{MLZ}$, and WST have made substantive intellectual contributions to this study, substantial contributions to the conception and design of it as well as to the acquisition, analysis, and interpretation of data. All of them have been also involved in the drafting and revision of the manuscript. All authors have contributed to and seen the manuscript. All authors read and approved the final manuscript.

\section{Acknowledgements}

This study was financially supported by the open Project Funding of State Key Laboratory of Bioreactor Engineering of China and the National High Technology Research and Development Program of China (No.

2007AA060904 and No. 2012AA061503).

Received: 5 September 2014 Accepted: 10 November 2014

Published online: 19 November 2014

\section{References}

1. Yong P, Rowson NA, Farr JPG, Harris IR, Macaskie LE (2002) Bioreduction and biocrystallization of palladium by Desulfovibrio desulfuricans NCIMB 8307. Biotechnol Bioeng 80:369-379

2. Hennebel T, De Gusseme B, Boon N, Verstraete W (2009) Biogenic metals in advanced water treatment. Trends Biotechnol 27:90-98

3. Rotaru AE, Jiang W, Finster K, Skrydstrup T, Meyer RL (2012) Non-enzymatic palladium recovery on microbial and synthetic surfaces. Biotechnol Bioeng 109:1889-1897

4. Karthikeya S, Beveridge TJ (2002) Pseudomonas aeruginosa biofilms react with and precipitate toxic soluble gold. Environ Microbiol 4:667-675

5. Humphries AC, Nott KP, Hall LD, Macaskie LE (2005) Reduction of Cr(VI) by immobilized cells of Desulfovibrio vulgaris NCIMB 8303 and Microbacterium sp. NCIMB 13776. Environ Microbiol 90:589-596

6. Lloyd JR, Yong P, Macaskie LE (1998) Enzymatic recovery of elemental palladium by using sulfate-reducing bacteria. Appl Environ Microbiol 64:4607-4609

7. Creamer NJ, Mikheenko IP, Yong P, Deplanche K, Sanyahumbi D, Wood J, Pollmann K, Merroun M, Selenska-Pobell S, Macaskie L (2007) Novel supported Pd hydrogenation bionanocatalyst for hybrid homogeneous/ heterogeneous catalysis. Catal Today 128:80-87

8. De Windt W, Aelterman P, Verstraete W (2005) Bioreductive deposition of palladium (0) nanoparticles on Shewanella oneidensis with catalytic activity towards reductive dechlorination of polychlorinated biphenyls. Environ Microbiol 7:314-325

9. Deplanche K, Caldelari I, Mikheenko IP, Sargent F, Macaskie LE (2010) Involvement of hydrogenases in the formation of highly catalytic Pd (0) nanoparticles by bioreduction of Pd (II) using Escherichia coli mutant strains. Microbiol 156:2630-2640

10. Martins M, Assuncao A, Martins H, Matos AP, Costa MC (2013) Palladium recovery as nanoparticles by an anaerobic bacterial community. J Chem Technol Biotechnol 88:2039-2045

11. Chidambaram D, Hennebel T, Taghavi S, Mast J, Boon N, Verstraete W, van der Lelie D, Fitts JP (2010) Concomitant microbial generation of palladium nanoparticles and hydrogen to immobilize chromate. Environ Sci Technol 44:7635-7640

12. Niu K, Zhang $X$, Tan WS, Zhu ML (2010) Characteristics of fermentative hydrogen production with Klebsiella pneumoniae ECU-15 isolated from anaerobic sewage sludge. Int J Hydrogen Energy 35:71-80

13. Hennebel T, Van Nevel S, Verschuere S, De Corte S, De Gusseme B, Cuvelier C, Fitts JP, Van der Lelie D, Boon N, Verstraete W (2011) Palladium nanoparticles produced by fermentatively cultivated bacteria as catalyst for diatrizoate removal with biogenic hydrogen. Appl Microbiol Biotechnol 91:1435-1445

14. Deplanche K, Merroun ML, Casadesus M, Tran DT, Mikheenko IP, Bennett JA, Zhu J, Jones IP, Attard GA, Swlenska-Pobell S, Macaskie LE (2012) Microbial synthesis of core/shell gold/palladium nanoparticles for applications in green chemistry. J R Soc Interface 9:1705-1712

15. Han GZ, Chen MD (2008) Microwave peak absorption frequency of liquid. S Sci China Ser G-Phys Mech Astron 51:1254-1263

16. Tzima E, Irani-Tehrani M, Kiosses WB, Dejana E, Schultz DA, Engelhardt B, Cao G, DeLisser H, Schwartz MA (2005) A mechanosensory complex that mediates the endothelial cell response to fluid shear stress. Nature 437:426-431

17. Deplanche K, Mikheenko I, Bennett J, Merroun M, Mounzer H, Wood J, Macaskie $L$ (2011) Selective oxidation of benzyl-alcohol over biomasssupported Au/Pd bioinorganic catalysts. Top Catal 54:1110-1114

18. Bunge $M$, Sobjerg LS, Rotaru AE, Gauthier D, Lindhardt AT, Hause G, Finster K, Kingshott P, Skrydstrup T, Meyer RL (2010) Formation of palladium (0) nanoparticles at microbial surfaces. Biotechnol Bioeng 107:206-215

19. Wang RF, Wang H, Feng HQ, Ji S (2013) Palladium decorated nickel nanoparticles supported on carbon for formic acid oxidation. Int $J$ Electrochem Sci 8:6068-6076 
20. Tobin JM, White C, Gadd GM (1994) Metal accumulation by fungi: applications in environmental biotechnology. J Ind Microbiol 13:126-130

21. Gumbart JC, Beeby M, Jensen GJ, Roux B (2014) Escherichia coli peptidoglycan structure and mechanics as predicted by atomic-scale simulations. Plos Comput Biol 10:1003475

22. Nies DH (2003) Efflux-mediated heavy metal resistance in prokaryotes. FEMS Microbiol Rev 27:313-339

23. Hodgson L, Tarbell JM (2002) Solute transport to the endothelial intercellular cleft: the effect of wall shear stress. Ann Biomedl Eng 30:936-945

24. Silva-Santisteban BOY, Filho FM (2005) Agitation, aeration and shear stress as key factors in inulinase production by Kluyveromyces marxianus. Enzyme Microb Technol 36:717-724

25. Lantz J, Gardhagen R, Karlsson M (2012) Quantifying turbulent wall shear stress in a subject specific human aorta using large eddy simulation. Med Eng Phys 34:1139-1148

26. Allen JJ, Shockling MA, Kunkel GJ, Smits AJ (2007) Turbulent flow in smooth and rough pipes. Phil Trans R Soc A 365:699-714

27. Onishi R, Matsuda K, Takahashi K, Kurose R, Komori S (2008) Retrieval of collision kernels from the change of droplet size distributions with linear inversion. Phys Scripta 2008:014050

28. Klaewkla R, Arend M, Hoelderich WF (2011) A review of mass transfer controlling the reaction rate in heterogeneous catalytic systems. In: de Mass Transfer-Advanced Aspects. InTech, Germany, pp 668-684

29. Evans JR, Davids WG, MacRae JD, Amirbahman A (2002) Kinetics of cadmium uptake by chitosan-based crab shells. Water Res 36:3219-3226

30. Ghadge RS, Patwardhan AW, Joshi JB (2006) Combined effect of hydrodynamic and interfacial flow parameters on lysozyme deactivation in a stirred tank bioreactor. Biotechnol Prog 22:660-672

31. Cherry RS, Papoutsakis ET (1986) Hydrodynamic effects on cells in agitated tissue culture reactors. Bioproc Eng 1:29-41

doi:10.1186/s40643-014-0028-2

Cite this article as: Lei et al:: Effect of fluid shear stress on catalytic activity of biopalladium nanoparticles produced by Klebsiella Pneumoniae ECU-15 on $\mathrm{Cr}(\mathrm{VI})$ reduction reaction. Bioresources and Bioprocessing 2014 1:28.

\section{Submit your manuscript to a SpringerOpen ${ }^{\circ}$ journal and benefit from:}

- Convenient online submission

- Rigorous peer review

- Immediate publication on acceptance

- Open access: articles freely available online

- High visibility within the field

- Retaining the copyright to your article

Submit your next manuscript at $\gg$ springeropen.com 